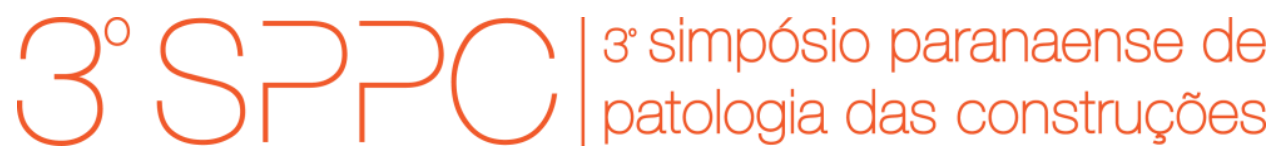

ISSN 2526-7248 artigo n. 3SPPC1014, pp. 156-165, 2018

\title{
Durabilidade de concretos com cimento supersulfatado: uma revisão da literatura
}

\author{
Pinto, Sabrina Requião ${ }^{1}$; Medeiros-Junior, Ronaldo A. ${ }^{2}$; Angulski da Luz, Caroline ${ }^{3}$ \\ ${ }^{1}$ Mestranda, Universidade Federal do Paraná, sabrinarequiao@yahoo.com.br \\ 2 Professor, Universidade Federal do Paraná, medeirosjunior.ufpr@gmail.com \\ 3 Professora, Universidade Tecnológica Federal do Paraná, angulski@hotmail.com
}

Resumo: A escória de alto-forno é um coproduto da indústria de ferro gusa que inicialmente não possui capacidade aglomerante mas, ao ser associada à outros materiais que promovam um alto $\mathrm{pH}$ da mistura, pode ser capaz de atuar como um material aglomerante. Atualmente, a principal destinação da escória de alto forno é a industria cimentícia para a produção de cimento Portland. Porém, é possível também o seu emprego na produção de cimentos com baixo ou nenhum teor de clínquer, como é o caso do cimento supersulfatado (CSS). O cimento supersulfatado em geral apresenta a seguinte composição: 80 a 90\% de escória de alto forno, 10 a $20 \%$ de sulfato de cálcio e 1 a $5 \%$ de ativador alcalino. Após um período de escassez de pesquisas, o cimento supersulfatado ressurge no cenário científico como uma promissora alternativa como material aglomerante de baixo impacto ambiental em substituição ao cimento Portland. Dentre os principais aspectos que demandam estudos, quando se trata de novos materiais, destaca-se o desempenho à durabilidade. Diante desse contexto, este artigo tem como objetivo apresentar uma revisão dos principais estudos acerca da durabilidade de concretos produzidos com cimento supersulfatado.

Palavras-chave: Escória de alto forno, Cimento supersulfatado, Durabilidade, Cimento de baixo impacto ambiental.

Abstract: Blast furnace slag is a co-product from iron industry and has no binding properties.
However, if it combined with other materials that promote a high pH of the mixture, it may be able
binding capacity. Nowadays, the main destination of blast furnace slag is the cement industry for
Portland cement production. However, it is also possible to use it in the production of no-clinker
cements such as supersulfated cement (SSC). The supersulfated cement shows the composition: 80
to $90 \%$ blast furnace slag, 10 to $20 \%$ calcium sulfate and 1 to $5 \%$ alkaline activator. After a shortage
of research, supersulfated cement return in the science landscape as a promising alternative as an
environmentally friendly cement replacing the Portland cement. One of the main aspects that require
studies, when it comes to new materials, is the durability performance. In this context, this article
aims to present a review of the main studies on the durability of concrete produced with supersulfated
cement.

Keywords: Blast furnance slag, Supersulfated cement, Durability, Environmentally friendly cement. 
PINTO, S. R.; MEDEIROS-JUNIOR, R. A.; ANGULSKI DA LUZ, C., DURABILIDADE DE CONCRETOS COM CIMENTO SUPERSULFATADO: UMA REVISÃO DA LITERATURA. $3^{\circ}$ Simpósio Paranaense de Patologia das Construções ( $3^{\circ}$ SPPC), artigo 3SPPC1014, pp. 156 - 165, 2018. DOI: 10.4322/2526-7248.014

\section{Introdução}

O cimento supersulfatado (CSS) foi desenvolvido e posteriormente patenteado em 1908 pelo químico alemão Hans Kühl. As primeiras produções ocorreram em 1914 na Alemanha e em 1932 na França e Bélgica [1]. Seu desenvolvimento baseou-se na descoberta de que as propriedades hidráulicas das escórias de alto-forno solidificadas em estado vítreo poderiam ser ativadas tanto por materiais básicos, tal como o hidróxido de cálcio, como também por materiais ácidos, como a gipsita [2]. Após a Segunda Guerra mundial, houve um aumento em sua aplicação devido à escassez de clínquer de Cimento Portland [3]. Entretanto, registrou-se o seu desaparecimento no mercado, motivado a princípio pela alteração da composição química da escória, especificamente dos teores de $\mathrm{Al}_{2} \mathrm{O}_{3}$, ocasionada pela mudança das matérias primas empregadas nos processos siderúrgicos [4,5]. Nos últimos anos os estudos acerca do cimento supersulfatado foram aos poucos retomados, incentivados em especial pelo seu baixo impacto ambiental. Os principais documentos vigentes atualmente que normatizam a produção de cimento supersulfatado são as normas europeia EN 15743/2010 [6] e indiana IS 6909/1990 [7].

A produção do cimento supersulfatado consiste na mistura de escória de alto forno, sulfato de cálcio e um ativador alcalino. O sulfato de cálcio pode ser encontrado na forma dihidratado $\left(\mathrm{CaSO}_{4} .2 \mathrm{H}_{2} \mathrm{O}\right)$, hemihidrato $\left(\mathrm{CaSO}_{4} .1 / 2 \mathrm{H}_{2} \mathrm{O}\right)$, ou anidrita (sulfato de cálcio anidro, $\mathrm{CaSO}_{4}$ ). Há também a possibilidade do emprego de resíduos industrias como o fosfogesso e o fluorgesso, oriundos das industrias produtoras de ácido fosfórico e ácido hidrofluórico, respectivamente. O ativador alcalino mais empregado é o cimento Portland, porém o hidróxido de potássio $(\mathrm{KOH})$, hidróxido de cálcio $\left(\mathrm{Ca}(\mathrm{OH})_{2}\right)$ e carbonato de sódio $\left(\mathrm{Na}_{2} \mathrm{CO}_{3}\right)$ também podem ser citados como exemplos de ativadores [8].

Com o início da hidratação, os principais compostos formados são a etringita e o silicato de cálcio hidratado (C-S-H). Outros produtos também pode ser encontrados, em menores quantidades, como gipsita $\left(\mathrm{CaSO}_{4} .2 \mathrm{H}_{2} \mathrm{O}\right)$ e hidrotalcita

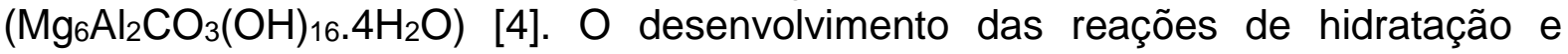
consequentemente do desempenho das propriedades do material no estado endurecido estão amplamente associadas à composição e características da escória de alto forno e do sulfato de cálcio, assim como do ativador empregado. Angulski da Luz e Hooton [9] compararam o processo de hidratação de cimentos supersulfatados compostos com escórias com baixo e alto teor de alumina, sob diferentes temperaturas de cura. Os autores identificaram que o CSS composto por escória com alto teor de alumina promove maiores resistências mecânicas e é melhor influenciado pela temperatura de cura. Rubert et al. [10], ao avaliarem o efeito do tipo $\left(\mathrm{KOH}\right.$ e $\left.\mathrm{Ca}(\mathrm{OH})_{2}\right)$ e teor $(0,2 ; 0,5$; e $0,8 \%)$ de ativador empregado sobre a hidratação do CSS, concluíram que ambos os ativadores promoveram um ganho de resistência considerável. Por outro lado, a relação entre aumento de resistência e aumento do teor de ativador foi proporcionalmente direta apenas para a ativação por $\mathrm{Ca}(\mathrm{OH})_{2}$. Em se tratando de ativação com cimento Portland, inicialmente certos autores observaram que ocorria um efeito negativo no desenvolvimento da resistência mecânica com o aumento do teor empregado (2\% para 5\%) [11]. Porém, recentemente, Jain e Garg [12] estudaram 3 teores de ativação com cimento Portland (5, 7 e 10\%) e observaram um ganho de resistência 
PINTO, S. R.; MEDEIROS-JUNIOR, R. A.; ANGULSKI DA LUZ, C., DURABILIDADE DE CONCRETOS COM CIMENTO SUPERSULFATADO: UMA REVISÃO DA LITERATURA. $3^{\circ}$ Simpósio Paranaense de Patologia das Construções (3० SPPC), artigo 3SPPC1014, pp. 156 - 165, 2018. DOI: 10.4322/2526-7248.014

entre o primeiro e segundo teores, seguido por um decréscimo ao aumentar para $10 \%$.

Apesar de não haver registros de aplicações no Brasil, na literatura internacional o emprego de cimentos supersulfatados mostra-se vantajoso principalmente em estruturas expostas tanto à água do mar quanto à águas contaminadas por sulfatos [2, 13-14]. Wang et al. 2016 [15] investigaram o uso de cimento supersulfatado em concretos massa e observaram menores picos de calor de hidratação $\left(52,5^{\circ} \mathrm{C}\right)$, em relação ao cimento Portland $\left(78^{\circ} \mathrm{C}\right)$. Portanto o CSS mostra-se também uma solução válida para mitigar a ocorrência de fissuras de origem térmica. Vale destacar a existência de CSS desenvolvido comercialmente, como é o caso austríaco do Slagstarß A Figura 1 apresenta a construção de uma estação de tratamento de esgoto, na cidade de Wildon na Áustria, na qual se empregou o cimento supersulfatado Slagstar ${ }^{\circledR}$. Com um consumo de cimento de 380 a 450 $\mathrm{kg} / \mathrm{m}^{3}$ e relação água/cimento de 0,37 a 0,44 foi possível atingir uma resistência à compressão em torno de $80 \mathrm{MPa}[16]$.

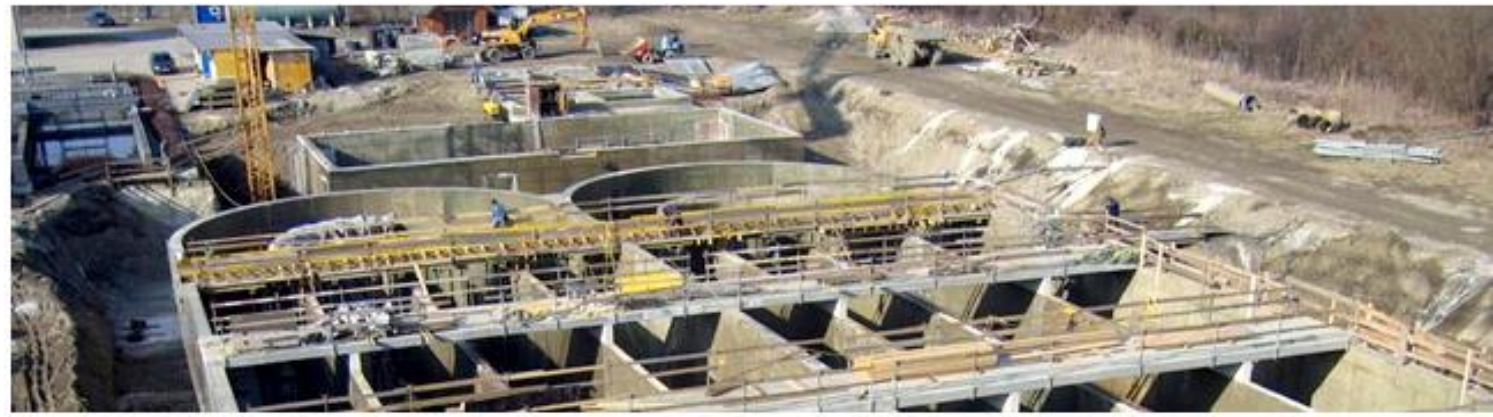

Figura 1: Estação de tratamento construída com cimento supersulfatado (Slagstar®) na cidade de Wildon, Áustria [16].

Na Figura 2 é apresentado outro caso de aplicação do CSS Slagstar®, desta vez na construção de silos de cimento [2]. Do ponto de vista estético, o resultado proporcionado pelo uso do CSS (à direita, Figura 2) apresenta maior qualidade e um tom mais claro, comparado ao cimento Portland (à esquerda, Figura 2).

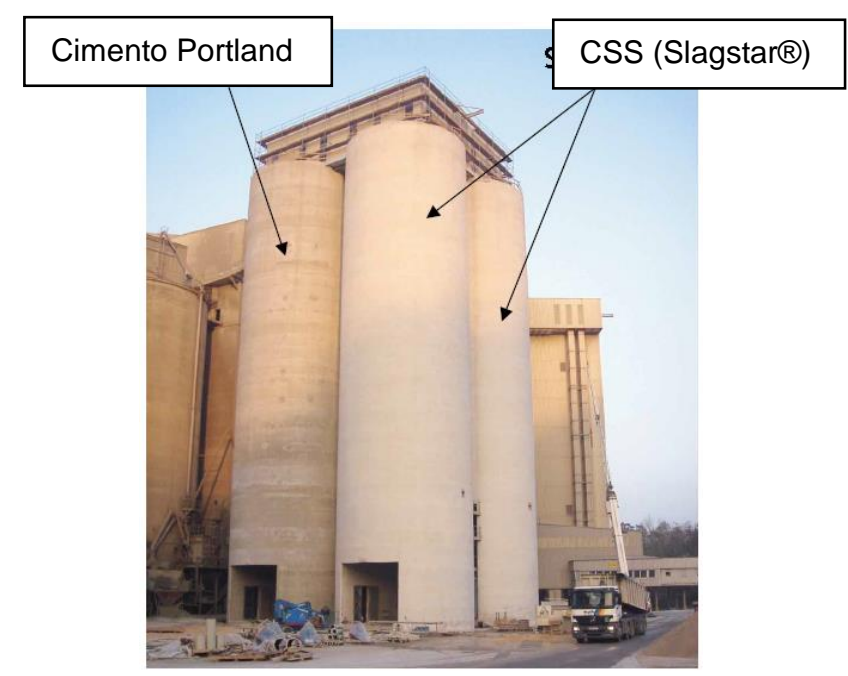

Figura 2: Silos de cimento construídos com cimento Portland, à esquerda, e cimento supersulfatado (Slagstar®), à direita, Áustria [2]. 
PINTO, S. R.; MEDEIROS-JUNIOR, R. A.; ANGULSKI DA LUZ, C., DURABILIDADE DE CONCRETOS COM CIMENTO SUPERSULFATADO: UMA REVISÃO DA LITERATURA. $3^{\circ}$ Simpósio Paranaense de Patologia das Construções (30 SPPC), artigo 3SPPC1014, pp. 156 - 165, 2018. DOI: 10.4322/2526-7248.014

Por se tratar de uma tecnologia cimentícia ainda pouco explorada, principalmente no Brasil, os estudos das propriedades e desempenho do CSS tornam-se necessárias para estimar o seu comportamento e vida útil quando empregado em estruturas reais. Diante disso, o presente artigo apresenta uma breve revisão dos principais estudos existentes sobre durabilidade de materiais cimentícios com cimento supersulfatado. Pretende-se assim, disponibilizar de forma compilada o estado da arte das pesquisas sobre o CSS aos atuais pesquisadores do tema, assim como também fomentar o desenvolvimento de novas pesquisas acerca do cimento supersulfatado nacionalmente.

\section{Durabilidade}

A Tabela 1 apresenta em ordem cronológica as propriedades avaliadas e relações água/cimento adotadas nos principais estudos sobre durabilidade de matrizes cimentícias com cimento supersulfatado.

Tabela 1: Propriedades e relações água/cimento avaliadas em estudos sobre a durabilidade de argamassas e concretos com CSS.

Ref. Ano Rel. a/c

Propriedades avaliadas

\begin{tabular}{|c|c|c|c|}
\hline [17] & 1961 & 0,60 & Ataque por sulfatos e ácido sulfúrico \\
\hline [11] & 1981 & 0,48 & Desgaste superficial \\
\hline [14] & 1981 & 0,43 & Carbonatação \\
\hline [18] & 1985 & 0,27 & Ataque por sulfatos e carbonatação \\
\hline [19] & 1988 & 0,27 & Carbonatação \\
\hline [20] & 1993 & 0,28 & Reação álcali agregado \\
\hline [21] & 1999 & 0,50 & $\begin{array}{l}\text { Permeabilidade ao ar, impermeabilidade à água, penetração } \\
\text { de íons cloreto, carbonatação e resistência ao gelo e degelo }\end{array}$ \\
\hline [22] & 2003 & * & Ataque por sulfatos \\
\hline [13] & 2003 & 0,27 & Ataque por sulfatos \\
\hline [23] & 2011 & * & Ataque por sulfatos e carbonatação \\
\hline [24] & 2012 & 0,50 & $\begin{array}{l}\text { Retração, ataque por sulfatos, carbonatação, penetração de } \\
\text { íons cloretos e absorção de água }\end{array}$ \\
\hline [25] & 2015 & 0,48 & Ataque por sulfatos \\
\hline [26] & 2015 & 0,50 & Ataque por sulfatos \\
\hline [27] & 2016 & * & $\begin{array}{l}\text { Ataque por soluções agressivas (água marinha natural e } \\
\text { sulfatos) e corrosão da armaduras }\end{array}$ \\
\hline [28] & 2016 & 0,35 & $\begin{array}{l}\text { Carbonatação, penetração de íons cloreto e absorção de } \\
\text { água }\end{array}$ \\
\hline [29] & 2016 & 0,60 & Ataque por sulfatos \\
\hline [30] & 2017 & * & Penetração de íons cloreto e corrosão da armaduras \\
\hline
\end{tabular}

*Dados não apresentados.

Como pode ser observado na Tabela 1, há uma maior concentração de estudos que avaliam o ataque por sulfatos e que adotam baixas relações água/cimento. A seguir são apresentados características, resultados e conclusões sobre estudos a respeito da áção de mecanismos de degradação (ataque por sulfatos, carbonatação, 
PINTO, S. R.; MEDEIROS-JUNIOR, R. A.; ANGULSKI DA LUZ, C., DURABILIDADE DE CONCRETOS COM CIMENTO SUPERSULFATADO: UMA REVISÃO DA LITERATURA. $3^{\circ}$ Simpósio Paranaense de Patologia das Construções (30 SPPC), artigo 3SPPC1014, pp. 156 - 165, 2018. DOI: 10.4322/2526-7248.014

penetração por íons cloretos e ação do gele e degelo) em argamassas e concretos produzidos com cimento supersulfatado.

\subsection{Ataque por sulfatos}

A ação de sulfatos presentes em águas ou solos contaminados pode comprometer seriamente a durabilidade das estruturas de concreto. Em relação ao CSS, verificase na literatura que há um considerável entendimento sobre como este mecanismo de ação ocorre nas matrizes de cimentícias compostas por cimento supersulfatado. Um dos primeiros estudos neste tema que se tem registro, foi realizado no Central Building Research Institute (CBRI), na Índia, pelos pesquisadores Chopra e Lal [17]. Em uma primeira etapa, os autores buscaram encontrar a melhor composição de cimento supersulfatado variando a composição de escória (80 e 75\%), sulfato de cálcio $(7,5 ; 10 ; 15$ e 17,5\%) e cimento Portland $(2,5 ; 5 ; 7,5$ e 10\%). A proporção que promoveu melhor desempenho mecânico foi de 75:20:5, motivada possivelmente pelas características de baixas relações $\mathrm{CaO} / \mathrm{SiO}_{2}$ e $\mathrm{SiO}_{2} / \mathrm{Al}_{2} \mathrm{O}_{3}$ apresentadas pela escória empregada. Na sequência, ao avaliar parâmetros de durabilidade, verificouse uma expansão de $0,025 \%$ em amostras de argamassa imersas em solução de sulfato de sódio (5\%), durante um ano, indicando assim uma boa resistência à ambientes com sulfatos. Além disso, foi investigada a resistência à compressão de cubos de argamassa, compostos por CSS e cimento Portland (CP) após a exposição ao longo de 1 ano em diferentes soluções. A partir da Tabela 2, que mostra os resultados obtidos, é possível destacar a maior suscetibilidade do CSS ao ataque por águas ácidas mas boa resistência à águas marinhas.

Tabela 1: Resistência à compressão de amostras cúbicas de argamassa após 1 ano de exposição em diferentes soluções [17].

\begin{tabular}{ccccc}
\hline \multicolumn{6}{c}{ Resistência à compressão após 1 ano de imersão (MPa) } \\
\hline $\begin{array}{c}\text { Água } \\
\text { destilada }\end{array}$ & $\begin{array}{c}\text { Solução: } \mathrm{KCl}(1 \%) \\
+\mathrm{NaCl}(3 \%)\end{array}$ & $\begin{array}{c}\text { Água destilada } \\
\text { saturada com } \mathrm{CO}_{2}\end{array}$ & $\begin{array}{c}\text { Solução: } \\
\mathrm{H}_{2} \mathrm{SO}_{4}(2 \%)\end{array}$ \\
\hline $\mathrm{CP}$ & 47 & 40 & 40,4 & 27,7 \\
\hline CSS & 89,9 & 85,8 & 74,6 & 35,8 \\
\hline
\end{tabular}

Grounds [18] ao estudar o ataque por sulfatos de cálcio, sódio e magnésio em pastas cimentícias concluiu que o CSS apresenta adequada resistência frente aos dois primeiros tipos de sulfatos. Porém, em relação ao sulfato de magnésio houve rápida decomposição das amostras e dessa forma o autor sugere que o mecanismo de ataque não ocorre da mesma maneira que em matrizes de cimento Portland e por isso há maior expansão e desintegração do CSS. O bom desempenho ao ataque por sulfatos, principalmente de sódio, vem sendo reafirmado por diversos autores [13, 22, 23, 27 e 29]. Ioannou [24] realizou um estudo aprofundado sobre o desempenho de cimentos supersulfatados e sulfoaluminatos. $O$ autor conclui que ambos os cimentos são resistentes ao ataque por sulfatos principalmente pois, diferentemente do cimento Portland, nestes os aluminatos já estão combinados em compostos sulfoaluminatos e há menor disponibilidade de portlandita $(\mathrm{Ca}(\mathrm{OH}) 2)$, produtos necessários para as reações expansivas. Resultados obtidos por Varella [29] indicam que as características e proporções de matérias que compõem o CSS também pode ser um fator no desempenho à durabilidade. Enquanto amostras compostas por gipsita apresentaram expansões dimensionais dentro do limite 
PINTO, S. R.; MEDEIROS-JUNIOR, R. A.; ANGULSKI DA LUZ, C., DURABILIDADE DE CONCRETOS COM CIMENTO SUPERSULFATADO: UMA REVISÃO DA LITERATURA. $3^{\circ}$ Simpósio Paranaense de Patologia das Construções (30 SPPC), artigo 3SPPC1014, pp. 156 - 165, 2018. DOI: 10.4322/2526-7248.014

recomendado na literatura, as amostras produzidas com fosfogesso ultrapassaram tal limite, ou seja, não foram consideradas resistentes à sulfato.

\subsection{Carbonatação}

A ação do dióxido de carbono $\left(\mathrm{CO}_{2}\right)$ na matriz cimentícia do CSS difere-se substancialmente quando comparado com o cimento Portland. Conforme Majumdar et al. [14] explicam, a difusão do $\mathrm{CO}_{2}$ no cimento supersulfatado promove a decomposição da etringita, possível contribuinte na resistência mecânica. Em seu estudo experimental, os autores verificaram maior efeito da carbonatação em matrizes reforçadas com fibra de vidro ao empregar o CSS ao invés do cimento Portland. Em contrapartida, os autores conseguiram amenizar tal efeito com a adoção de camadas protetoras de argamassa. Nesse sentido, loannou [24], que também encontrou baixas resistências à carbonatação em seu estudo, destaca que a questão da baixa alcalinidade das matrizes se torna mais relevante do que outros fatores, como a relação água/cimento, no que tange a carbonatação em matrizes de cimento supersulfatado. Uma alternativa que foi estudada para reduzir essa deficiência foi o emprego de formas pré fabricadas que promovem uma melhor qualidade superficial do concreto [28]. Conforme apresentado na Figura 3, foi possível uma redução aproximadamente de 35\% da profundidade de carbonatação.

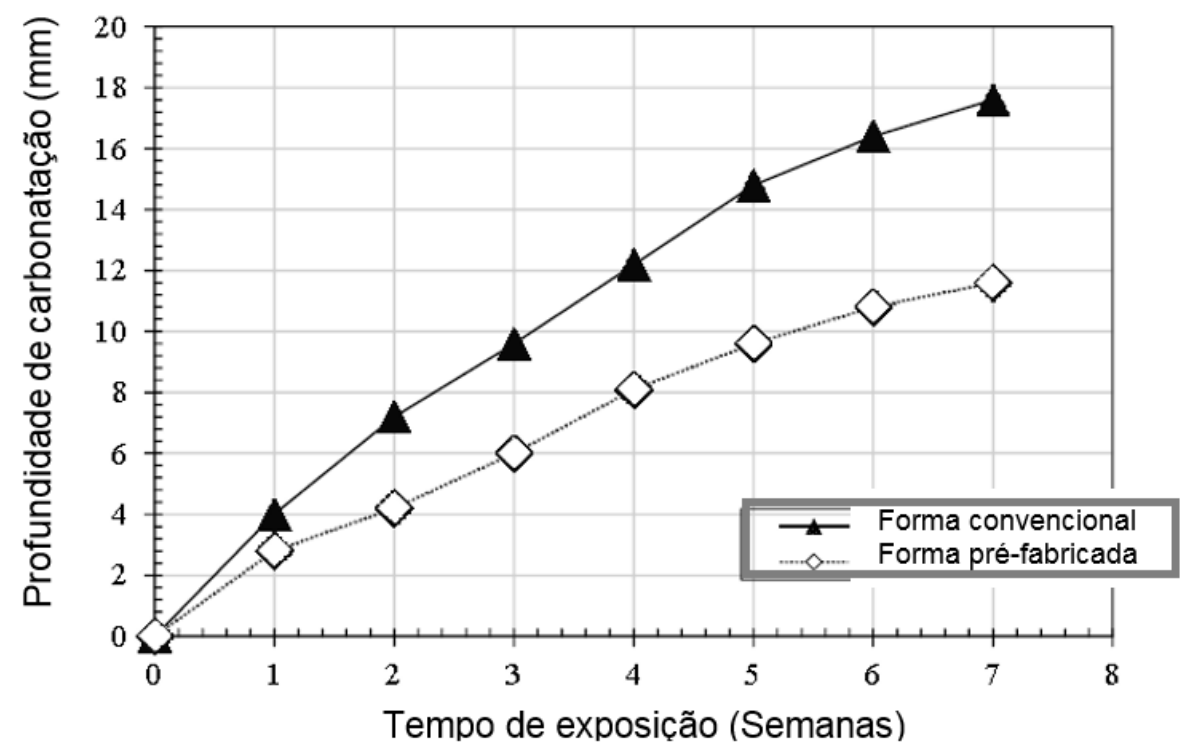

Figura 3: Profundidade de carbonatação em função do tipo de forma adotada (Adaptado e traduzido) [28].

\subsection{Penetração de íons cloreto}

A resistência ao ingresso de íons cloreto é uma das propriedades essencias à estruturas expostas à água ou atmosfera marinhas. Diante disso, muito estudos tem por objetivo aprimorar a qualidade do concreto tal como com o uso de adições minerais. Já é bem compreendido os efeitos positivos causados pelo emprego de escória de alto forno na estrutura e tamanho dos poros de concretos com cimento Portland. Guneyisi e Gesoglu [31] investigaram a durabilidade de concretos com altos teores de escória de alto forno (50 a 80\%) e observaram que houve um expressivo decréscimo em propriedades como a absorção de água e penetração de 
PINTO, S. R.; MEDEIROS-JUNIOR, R. A.; ANGULSKI DA LUZ, C., DURABILIDADE DE CONCRETOS COM CIMENTO SUPERSULFATADO: UMA REVISÃO DA LITERATURA. $3^{\circ}$ Simpósio Paranaense de Patologia das Construções (30 SPPC), artigo 3SPPC1014, pp. 156 - 165, 2018. DOI: 10.4322/2526-7248.014

íons cloreto à medida que maiores teores de escória foram adicionados. Conforme resultados obtidos por Thomas et al. [32], a maior resistência à penetração de íons cloreto está associada a maior capacidade de fixação dos íons pela presença de quantidades significativas de aluminatos.

Considerando que a escória é um dos principais materiais que compõem o CSS, e em geral, em elevados teores, suspeita-se que concretos com CSS apresentem um comportamento semelhante aos concretos com altos teores de escória, conforme exposto. loannou [24] também contemplou em seu estudo experimental a estimativa do coeficiente de difusão aparente de íons cloretos em diferentes tipos de cimento (CEM I, equivalente ao CP I; CEM III/A, equivalente ao CP III; CSS; CSS Slagstar; e cimento sulfoaluminato) e relações água/cimento $(0,35 ; 0,50 ; 0,65)$, conforme apresentado na Figura 4. Os resultados indicam que, exceto para a a/c igual a 0,35, os cimentos supersulfatados se mostram menores coeficientes de difusão em comparação ao cimento CEM I.

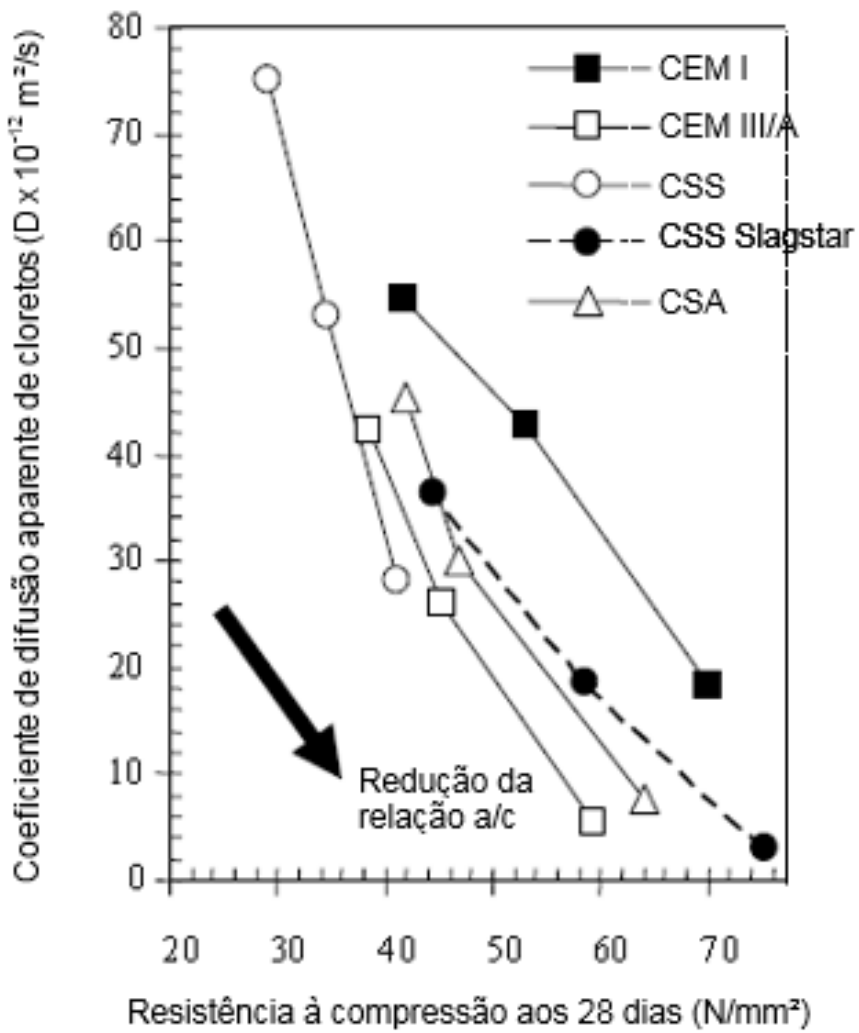

Figura 4: Coeficiente de difusão aparente de cloretos em função do tipo de cimento e relação a/c (Adaptado e traduzido) [24].

Nguyen et al. [30] identificaram consideráveis taxas de íons cloretos fixos em relação a quantidade de íons cloretos totais, confirmando o hipótese da boa capacidade de fixação de íons cloreto pela matriz cimentícia de CSS. Novak e Sommer [33] estudaram a ação de cloretos em amostras de concreto armado em cimentos CEMII/A-L (equivalente ao CP II F) e CSS. Os autores identificaram o início do processo de corrosão aos 25 dias nas amostras de CEMII/A-L. Por outro lado, nas amostras de CSS a frente de íons cloreto não ultrapassou a espessura de cobrimento até o final previsto para o teste, 132 dias. 
PINTO, S. R.; MEDEIROS-JUNIOR, R. A.; ANGULSKI DA LUZ, C., DURABILIDADE DE CONCRETOS COM CIMENTO SUPERSULFATADO: UMA REVISÃO DA LITERATURA. $3^{\circ}$ Simpósio Paranaense de Patologia das Construções ( $3^{\circ}$ SPPC), artigo 3SPPC1014, pp. 156 - 165, 2018. DOI: 10.4322/2526-7248.014

\subsection{Reação álcali agregado}

Concretos com cimento supersulfatado tendem a apresentar boa resistência à reação álcali agregado [20,33]. Min e Mingshu [20] investigaram a variação dimensional em barras de concreto com agregados inócus e reativos e cimentos supersulfatado e sulfoaluminato. Após 400 dias, os autores observaram que a expansão das amostras com diferentes agregados foram semelhantes, demonstrando assim a capacidade de mitigação de ambos os cimentos. Comportamento semelhante foi observado por Novak e Sommer [33].

\section{Considerações Finais}

O cimento supersulfatado mostra-se como uma nova alternativa de aglomerante com menor energia de produção e portanto mais susténtável. Entretanto, suas vantagens ambientais não dispensam uma análise acerca do seu comportamento ao longo do seu ciclo de vida, ou seja, um estudo da sua durabilidade. Verifica-se que há muitas questões a serem contempladas em estudos futuros, visto que as propriedades promovidas estão intimamente ligadas às características químicas e físicas dos materiais empregados, que variam em função da sua origem. Seu uso é muito promissor em estruturas que exigam maior controle do calor de hidratação (como barragens e fundações), em estruturas expostas à ambientes agressivos com a presença de sulfatos e águas marinhas, e por fim, em aplicações que priorizam o uso de materias mais sustentáveis (obtenção de selos verdes). No contexto nacional, somente a partir de um maior incentivo à pesquisas sobre o tema é que favorecerá um futuro encaminhamento para normalizações e a efetiva aplicação do CSS no país.

\section{Agradecimentos}

Os autores agradecem o apoio da Coordenação de Aperfeiçoamento de Pessoal de Nível Superior (CAPES) e do Programa de Pós-Graduação em Engenharia de Construção Civil da Universidade Federal do Paraná (PPGECC/UFPR).

\section{Referências}

[1] Aïtcin, P. C. (2008) Binders for durable and sustainable concrete. Modern concrete technology 16. Taylor and Francis Inc., New York, USA.

[2] Woltron, G. (2009) The Utilisation of GGBFS for Advanced Supersulfated. World Cement Magazine, 157-162.

[3] Juenger, M. C. G. et al. (2011) Advances in alternative cementitious binders. Cement and Concrete Research, v. 41, n. 12, p. 1232-1243.

[4] Gruskovnjak, A. et al. (2008) Hydration mechanisms of super sulphated slag cement. Cement and Concrete Research, 38(7), 983-992.

[5] Novak, R. et al. (2005) New knowledge regarding the supersulphated cement Slagstar. Material Science, v. 58, n.12, p. 70-78.

[6] EN 15743 (2010) Supersulfated Cement-Composition, Specification and Conformity Criteria, European Committee for Standardization (CEN), Brussels, Belgium. 
PINTO, S. R.; MEDEIROS-JUNIOR, R. A.; ANGULSKI DA LUZ, C., DURABILIDADE DE CONCRETOS COM CIMENTO SUPERSULFATADO: UMA REVISÃO DA LITERATURA. $3^{\circ}$ Simpósio Paranaense de Patologia das Construções (30 SPPC), artigo 3SPPC1014, pp. 156 - 165, 2018. DOI: 10.4322/2526-7248.014

[7] IS 6909 (1990) Specification for super sulphated cement. Bureau of Indian Standards, New Delhi, India.

[8] Juenger, M. C. G. et al. (2011) Advances in alternative cementitious binders. Cement and Concrete Research, v. 41, n. 12, p. 1232-1243.

[9] Angulski Da Luz, C.; Hooton, R. D. (2015) Influence of curing temperature on the process of hydration of supersulfated cements at early age. Cement and Concrete Research, 77: 69-75.

[10] Rubert, S. et al. (2015) The influence of the content and type of alkali activator on the process of hydration of supersulfated cements (SSC). In: 14th International Congress on the Chemistry of Cement (ICCC 2015), Beijin, China.

[11] Bijen, J.; \& Niël, E. (1981). Supersulphated cement from blastfurnace slag and chemical gypsum available in the Netherlands and neighbouring countries. Cement and Concrete Research, 11(3), 307-322.

[12] Jain, N.; \& Garg, M. (2015). Formulation of Sulphate Resistant Super Sulphated Cement Using Fluorogypsum and Granulated Blast Furnace Slag. IOSR Journal of Mechanical and Civil Engineering, 12(3), 153-159.

[13] Grounds, T.; Nowell, D.; \& Wilburn, F. (2003). Resistance of supersulfated cement to strong sulfate solutions. Journal of thermal analysis and calorimetry, 72(1), 181-190.

[14] Majumdar, A. J.; Singh, B.; \& Evans, T. J. (1981). Glass fibre-reinforced supersulphated cement. Composites, 12(3), 177-183.

[15] Wang, S. et al. (2016) The Use of Supersulfated Cement (SSC) in Mass Concrete. In: MATEC Web of Conferences. EDP Sciences, p. 07033.

[16] Novak, R.; Schneider, W.; \& Lang, E. (2005). New knowledge regarding the supersulphated cement Slagstar. Zement-Kalk-Gips (ZKG INTERNATIONAL), 58(12), 70-78.

[17] Chopra, S. K.; Lal, K. (1961) The manufacture of supersulphated cement from indian slag. Indian Concrete Journal, 38, I14.

[18] Grounds, T. (1985). The stability and durability of supersulphated cement paste and their relationship to ettringite. Thesis (PhD). Hatfield Polytechnic, University of Hertfordshire, Hertfordshire, UK.

[19] Grounds, T.; Midgley, H. G.; \& Novell, D. V. (1988). Carbonation of ettringite by atmospheric carbon dioxide. Thermochimica Acta, 135, 347-352.

[20] Min, D., \& Mingshu, T. (1993). Measures to inhibit alkali-dolomite reaction. Cement and concrete research, 23(5), 1115-1120.

[21] Stark, J., \& Frohburg, U. (1999). Investigations with supersulfated cement. In Modern Concrete Materials: Binders, Additions and Admixtures: Proceedings of the International Conference Held at the University of Dundee, Scotland, UK.

[22] Singh, M., \& Garg, M. (2003). Behaviour of low heat sulphate resistant cement in aggressive media. Indian Journal of Engineering \& Material Sciences, v.10, pp. 219-222, India.

[23] loannou, S., Paine, K., \& Quillin, K. (2011). Resistance of supersulfated cement concrete to carbonation and sulfate attack. In: Patricios, N. and Alifragkis, S., 
PINTO, S. R.; MEDEIROS-JUNIOR, R. A.; ANGULSKI DA LUZ, C., DURABILIDADE DE CONCRETOS COM CIMENTO SUPERSULFATADO: UMA REVISÃO DA LITERATURA. $3^{\circ}$ Simpósio Paranaense de Patologia das Construções ( $3^{\circ}$ SPPC), artigo 3SPPC1014, pp. 156 - 165, 2018. DOI: 10.4322/2526-7248.014

eds. Construction. Athens, Greece: Athens Institute for Education and Research, pp. 293-305.

[24] loannou, S. (2012). An assessment of the performance of calcium sulfoaluminate and supersulfated cements for use in concrete. Doctoral dissertation, University of Bath, UK.

[25] Jain, N., \& Garg, M. (2015). Formulation of Sulphate Resistant Super Sulphated Cement Using Fluorogypsum and Granulated Blast Furnace Slag. IOSR Journal of Mechanical and Civil Engineering, 12(3), 153-159.

[26] Niu, Q.; Zhang, R. (2015) Effect of supersulphated cement on sulfate attack of cement mortar. In: International Conference on Advances in Energy and Environmental Science (ICAEES 2015), Guangzhou, China.

[27] Nguyen, K. S. (2016) Using super sulfated cement for concrete durability in marine environment. In: The 7th International Conference of Asian Concrete Federation "Sustainable concrete for now and the future", Hanoi, Vietnam.

[28] Ioannou, S.; Badr, A., Kostova, K.; Paine, K.; \& Ibell, T. (2016). Utilization of fabric formwork for improving the durability of concrete from supersulfated cement. Key Engineering Materials, v. 711, pp. 615-621.

[29] Varela, M. V. F. (2016) Comportamento do cimento supersulfatado ao ataque por sulfatos. 104 f. Trabalho de Conclusão de Curso (Bacharelado em Engenharia Civil) - Universidade Tecnológica Federal do Paraná, Pato Branco, Paraná, Brasil.

[30] Nguyen, K. S. et al. (2017) Chloride Binding Ability and Anti-corrosion Properties of Supersulfated Cement in Seawater/Sand Mixing Concrete. In: Congrès International de Géotechnique-Ouvrages-Structures, pp. 367-376, Springer, Singapore.

[31] Guneyisi, E.; Gesoglu, M. (2008) A study on durability properties of highperformance concretes incorporating high replacement levels of slag, Material and Structures, 41 479-493.

[32] Thomas, M. D. A.; Hooton, R. D.; Scott, A., \& Zibara, H. (2012). The effect of supplementary cementitious materials on chloride binding in hardened cement paste. Cement and Concrete Research, 42(1), 1-7.

[33] Novak, D.; Sommer, H. (2002). A new low-heat sulfate resistant binder for mass concrete, HPC and SCC. In: Challenges of Concrete Construction, v. 5, pp. 213-222, Sustainable Concrete Construction: Proceedings of the International Conference held at the University of Dundee, Scotland, UK. 